This item is the archived peer-reviewed author-version of:

\title{
A non-contact approach for PWV detection : application in a clinical setting
}

\section{Reference:}

Campo Adriaan, Heuten Hilde, Goovaerts Inge, Ennekens Guy, Vrints Christiaan, Dirckx Joris.- A non-contact approach for PWV detection : application in a clinical setting

Physiological measurement / Institute of Physical Sciences in Medicine - ISSN 0967-3334 - 37:7(2016), p. 990-1003 Full text (Publisher's DOI): http://dx.doi.org/doi:10.1088/0967-3334/37/7/990

To cite this reference: http://hdl.handle.net/10067/1356660151162165141 


\title{
A non-contact approach for PWV detection: application in a clinical setting
}

\author{
Adriaan Campo ${ }^{1,3}$, Hilde Heuten ${ }^{2}$, Inge Goovaerts ${ }^{2}$, Guy Ennekens ${ }^{2}$, \\ Christiaan Vrints ${ }^{2}$ and Joris Dirckx ${ }^{1}$
}

1: Laboratory of Biomedical Physics; Faculty of Science; University of Antwerp; Groenenborgerlaan 171 B-2020 Antwerp; Belgium; www.ua.ac.be/bimef

2: Departement of Cardiology; University Hospital Antwerp; Antwerp; Wilrijkstraat 10; 2650 Edegem; Belgium; http://www.uza.cardionet.be

3: Ultrasound Elasticity Imaging Laboratory; Department of Biomedical

Engineering; Columbia University; 630 West 168th Street New York 10032; NY; http://orion.bme.columbia.edu/ueil/

Email: adriaan.campo@ua.ac.be

\begin{abstract}
A need for screening methods for arteriosclerosis led to the development of several approaches to measure pulse wave velocity (PWV) being indicative of arterial stiffness. Carotidfemoral PWV (cfPWV) can be measured between common carotid artery (CCA) and femoral artery (FA) displaying the physiologically important stiffness of the conduit arteries. However, this measurement approach has several disadvantages, and a local PWV-measurement of CCA-stiffness has been proposed as an alternative in the past.

In the presented pilot study, laser Doppler vibrometry (LDV) is used to measure PWV locally in the CCA ( $\mathrm{PWV} \mathrm{LDV}_{\mathrm{L}}$ ) in 48 patients aged between 48 and 70, with known atherosclerotic arterial disease: stabilized coronary artery disease (CAD), cerebro-vascular disease (CVD) or peripheral artery disease (PAD). Additionally, cfPWV, CCA distensibility coefficient (DC), CCA intimamedia thickness (IMT), blood pressure (BP) and age were evaluated.

LDV is a valid method for local PWV-measurement. The method is potentially easy to use, and causes no discomfort to the patient. $\mathrm{PWV}_{\mathrm{LDV}}$ correlates with age $(\mathrm{R}=0.432 ; \mathrm{p}=0.002)$ as reported in related studies using other techniques, and measured values lay between 2.5 and $5.8 \mathrm{~m} / \mathrm{s}$, which is well in line with literature measures of local PWV in the CCA.

In conclusion, $\mathrm{PWV}$ LDV potentially is a marker for arterial health, but more research in a larger and more homogeneous patient population is mandatory. In future studies, blood velocity measurements should be incorporated, as well as a reference method such as pulse wave imaging (PWI) or magnetic resonance imaging (MRI).
\end{abstract}


Keywords: arteriosclerosis, Laser Doppler vibrometry, pulse wave velocity

\section{Introduction:}

\subsection{Arterial stiffness as a marker for cardiovascular health:}

Cardiovascular disease (CD) is the most common cause of death worldwide, and its prevalence is rising. An important factor in the etiology of CD is stiffening of the aorta. Every heartbeat, a pulse wave propagates through the arterial system. Arterial stiffness can be monitored by assessing velocity of this pulse wave - or pulse wave velocity (PWV). As an increased PWV comes with stiffer arteries, high aortic PWV-values are a predictor for CD and mortality. Therefore, aortic PWV is an important screening target. For assessing aortic PWV, a velocity measurement of the pressure wave is typically performed between the common carotid artery (CCA) in the neck, and the femoral artery (FA) in the groin, termed carotidfemoral PWV or cfPWV (Laurent et al. 2006).

However, this type of measurement is not free of problems due to various reasons (Hermeling et al. 2007; Huybrechts et al. 2011; Kimoto et al. 2003; Laurent et al. 2006). An increase of CCA-stiffness can be representative for increased stiffness of the aorta instead, and methods to assess CCA-stiffness noninvasively attract a lot of attention (Laurent et al. 2003).

\subsection{Assessment of CCA-stiffness: rationale, benefits and shortcomings:}

Due to its central position and extensive dilation, the CCA shares many features with the aorta. Several studies indicate that CCA-stiffness is representative for aortic stiffness. As a direct indication, it was shown that an increase in CCA-stiffness parallels changes in ventricular architecture (Boutouyrie et al. 1995; Toprak et al. 2009; Weisz et al. 2014), cardiovascular events and mortality (van Sloten et al. 2014; Leone et al. 2008). Aortic stiffness is shown to correlate to CCA-stiffness, in some studies even in a linear fashion (Nagai et al. 1999). As an indirect indication, both increases in elastic artery stiffness and CCAstiffness are related to age (Ahlgren et al. 1997; Benetos et al. 1993), renal failure (Blacher et al. 1998), hypertension (Liao et al. 1999) and many other factors.

It must be noted that careful interpretation of CCA-stiffness is needed, however, as aortic and CCAstiffness are not equal indices. Distensibility of the CCA is lower than the aorta in normal conditions (Nagai et al. 1999; Länne et al. 1994). Also, the aorta is a heterogeneous unit that is affected differently in age and disease according to the location (Tsamis et al. 2013). Finally, the aorta is more vulnerable to degeneration, so CCA-stiffness possibly lacks sensitivity in certain cases (Länne et al. 1994). This is reflected in the study of Paini et al. that showed that aortic stiffness and CCA-stiffness are concordant, except for patients with high blood pressure (BP) and/or diabetes. In these cases, the aorta stiffened more than the CCA with age and other cardiovascular risk factors (Paini et al. 2006).

However, there exist other rationalities for studying CCA-stiffness. Increased thickening of the intimamedia layer in the CCA-wall is believed to be an early compensatory mechanism related to arterial stiffening and an early marker of atherosclerosis, stroke and other cardiovascular events (van Popele et al. 2001; Tran et al. 2012; Tsivgoulis et al. 2006; Simon et al. 2010). Additionally, increased CCA-stiffness is related to altered perfusion of the brain (Hirata et al. 2010). Finally, the CCA is an easily accessible structure with ultrasound (US) imaging. In conclusion, a measurement method for CCA-stiffness is an attractive tool for screening, diagnosis and research. 


\subsection{Research design:}

In the current study, a non-invasive, non-contact, optical method for CCA-stiffness assessment using Laser Doppler vibrometry (LDV) is presented. In previous work, LDV (Cooper 1999) has been used to detect vibration velocity of the skin overlaying a vessel. This velocity is in direct relation to the change in pressure inside the superficial vessel (De Melis et al. 2008). By assessing skin velocity with LDV, it is possible to detect the pulse wave in the underlying vessel, and consequently detect PWV of a the CCA (ccaPWV) (Campo et al. 2014).

LDV is evaluated for ccaPWV-detection (from here on, ccaPWV is used to term local PWV in the CCA, while ccaPWV measured with LDV is specifically termed $\left.\mathrm{PWV}_{\mathrm{LDV}}\right)$ in 52 patients aged between 48 and 70, with known atherosclerotic arterial disease: stabilized coronary artery disease (CAD), cerebro-vascular disease (CVD) and peripheral artery disease (PAD). The method will be compared with other indices for arterial stiffness including cfPWV, CCA distensibility coefficient (DC), PWV derived from DC (PWV $\mathrm{DC})$, intima-media thickness (IMT); and with the most important determinants of arterial stiffness BP and age. We hypothesize naively that $P W V_{L D V}$ relates at least with part of the reference parameters.

\section{Materials and Methods:}

\subsection{Patients:}

The population consisted of 52 patients (aged between 48 and 70, male/female). Patients were included when suffering from atherosclerotic arterial disease, i.e. stabilized CAD, CVD or PAD. Exclusion criteria were a serious vascular event within the last 6 weeks, recent surgery, uncontrolled hypertension or atrial fibrillation or premature ventricular contraction. Participants signed an informed consent and the research protocol was approved by the hospital ethical committee (reference 13/50/509). Patients were required to withhold any intake of food, medication, drugs or drinks other than water for 4 hours until the onset of the measurements. Medical history and medication was documented. At the beginning and at the end of every measurement session, heart rate $(\mathrm{HR})$ and brachial systolic and diastolic $\mathrm{BP}\left(\mathrm{BP}_{\text {systolic-brachial }}\right.$ or $\mathrm{BP}_{\text {diastolic- }}$ brachial) were measured using a cuff manometer. For a summary of patient characteristics see Table 1. 
Table 1: Summary of Patient Characteristics

\section{Patient Characteristics}

\begin{tabular}{|c|c|c|}
\hline Parameter & \multicolumn{2}{|c|}{ Amount } \\
\hline Male/Female & \multicolumn{2}{|c|}{$40 / 12$} \\
\hline Smoking & \multicolumn{2}{|c|}{14} \\
\hline Hypertension & \multicolumn{2}{|c|}{35} \\
\hline $\mathrm{DM}$ & \multicolumn{2}{|c|}{9} \\
\hline Cholesterol & \multicolumn{2}{|c|}{40} \\
\hline CIHD/PVD/CVD & \multicolumn{2}{|c|}{28} \\
\hline Plaques & \multicolumn{2}{|c|}{34} \\
\hline Parameter & Min-Max & mean \pm SD \\
\hline Length $(\mathrm{cm})$ & $149-190$ & $172 \pm 10$ \\
\hline Age (years) & $48-69$ & $54 \pm 5$ \\
\hline Weight $(\mathrm{kg})$ & $58-116$ & $82 \pm 14$ \\
\hline $\operatorname{BMI}\left(\mathrm{kg} / \mathrm{m}^{2}\right)$ & $20-35$ & $28 \pm 4$ \\
\hline $\mathrm{BP}_{\text {systolic }}(\mathrm{mmHg})$ & $96-171$ & $133 \pm 17$ \\
\hline $\mathrm{BP}_{\text {diastolic }}(\mathrm{mmHg})$ & $55-99$ & $77 \pm 10$ \\
\hline IMT (mm) & $0.49-1.08$ & $0.68 \pm 0.10$ \\
\hline $\mathrm{BP}_{\text {systolic-CCA }}$ & $77-151$ & $115 \pm 15$ \\
\hline $\mathrm{BP}_{\text {diastolic-CCA }}$ & $67-113$ & $89 \pm 11$ \\
\hline $\mathrm{D}_{\text {systolic }}(\mathrm{mm})$ & $4.84-9.19$ & $6.49 \pm 0.96$ \\
\hline $\mathrm{D}_{\text {diastolic }}(\mathrm{mm})$ & $4.42-8.71$ & $5.95 \pm 0.94$ \\
\hline $\mathrm{DC}\left(10^{3} / \mathrm{Pa}\right)$ & $0.02-0.29$ & $0.07 \pm 0.04$ \\
\hline $\mathrm{PWV}_{\mathrm{DC}}(\mathrm{m} / \mathrm{s})$ & $1.9-6.5$ & $6.2 \pm 1.4$ \\
\hline $\operatorname{cfPWV}(\mathrm{m} / \mathrm{s})$ & $6.5-15.9$ & $9.2 \pm 1.9$ \\
\hline $\mathrm{PWV}_{\mathrm{LDV}}(\mathrm{m} / \mathrm{s})$ & $2.5-5.8$ & $4.7 \pm 0.7$ \\
\hline
\end{tabular}

Table 1: overview of patient characteristics. Abbreviations: SD (standard deviation); DM (diabetes mellitus); CIHD (chronic ischemic heart disease); PVD (peripheral vascular disease); CVD (cerebrovascular disease); $\mathrm{BMI}$ (body mass index); $\mathrm{BP}_{\text {systolic/diastolic }}$ (brachial systolic/diastolic $\mathrm{BP}$ ); $\mathrm{BP}_{\text {systolic- }}$ $\mathrm{CCA} /$ diastolic-CCA $\left(\mathrm{CCA}\right.$ systolic/diastolic BP); IMT (intima-media thickness); $\mathrm{D}_{\text {systolic/diastolic }}$ (systolic/diastolic diameter); DC (distensibility coefficient); $\mathrm{PWV}_{\mathrm{DC}}$ (PWV derived from DC); cfPWV (PWV measured with applanation tonometry); $\mathrm{PWV}_{\mathrm{LDV}}$ (PWV measured with LDV using the MA). 


\subsection{Data acquisition:}

2.2.1. LDV:

2.2.1.1. Experimental setup:

3 Polytec (Polytec, Waldbronn, Germany) LDV units of the type IVS 200 were mounted on a platform, mounted on a solid stand. LDV is a laser-based technique for non-contact measurement of velocity and displacement of a reflecting surface, here applied on the human skin. Its working principle is based on the Doppler Effect and interference of coherent light beams (Cooper 1999). Using mirrors, 3 parallel beams could be aimed on the skin. Patients laid down on a stretcher next to the LDV-setup. Using a custom-built plastic spacer, 3 small discs of reflective tape were applied to the skin with exact mutual distance of $1 \mathrm{~cm}$. Discs were prepared with an ordinary perforator, such that all discs had the same shape and area. Orientation of the platform and the mirrors was tuned carefully such that each beam hit a disc surface perpendicularly. The discs were aligned along the CCA, and were positioned directly above the artery and downstream from the bifurcation. To ascertain correct placement of the discs, anatomy of CCA, bifurcation and surrounding tissues was imaged with US echography a priori. The CCA-trajectory was marked with a marker pen, and subsequently used for application of the discs using the spacer. Velocity signals of 3 vibrometers and 1 electrocardiogram (ECG) amplifier were recorded with a National Instruments external data acquisition card with sample rate of 125000 samples/second at 16 bits bit-depth (National Instruments, Austin, Texas, USA). Measurement range of the vibrometers was $\pm 5 \mathrm{~V} / \mathrm{mm} / \mathrm{s}$.

During each LDV-measurement, patients were asked to hold as still as possible between an audible startand-stop cue and they were urged to avoid swallowing. In each patient, 4 LDV-measurements were performed, including 1 dummy measurement to compensate for undetected body motions post hoc. 1 recording sequence lasted for 10 seconds capturing at least 5 heartbeats in every measurement. Distance between measurement beams incident on the skin was determined with a tape-measure (see Figure 1). A sequence of 4 LDV measurements - including application of reflective patches - did not take more than 5 minutes. 


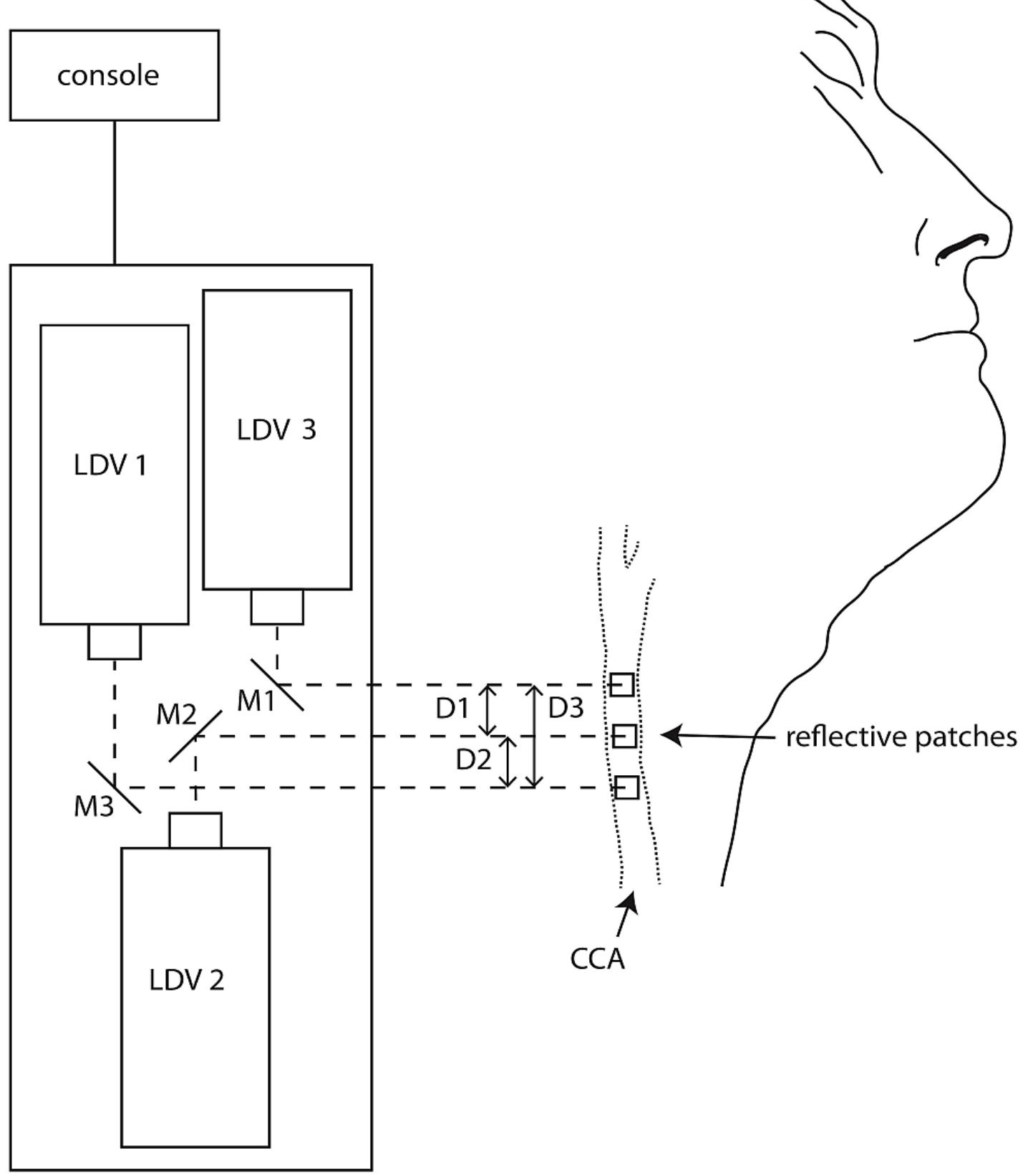

Figure 1: 3 laser Doppler vibrometer systems (LDV 1-3) mounted on a platform project 3 parallel low intensity laser beams by means of tunable mirrors (M1-3) on 3 equally spaced (D1-3, with D1=D2) reflective patches attached on a straight line along the trajectory of the right common carotid artery (CCA). 


\subsubsection{Data analysis:}

For the LDV-signals, pulse wave signals from every heart beat were filtered using a Savitsky-Golay polynomial filter with order 3 and window size of 751 points and overlap of $10 \%$, delivering filtered $0^{\text {th }}$, $1^{\text {st }}$ and $2^{\text {nd }}$ order derivatives of the initial signal representing respectively out-of-plane velocity, acceleration and jerk of the skin. All derivatives were filtered once with a low-pass Butterworth filter with order 4 and $1000 \mathrm{~Hz}$ cut-off frequency to remove remnant noise. This cut-off frequency was chosen because it removed the noise adequately without affecting the signal shape visibly. Subsequently, signals from separate heartbeats were isolated using the QRS-complex of the ECG-signal as a lead, allowing beatper-beat analysis of PWV. Finally, an automatic selection was done of signals of separate heartbeats, to remove truncated parts of the dataset due to spikes or unwanted movements. Per patient, a time window relative to the QRS-complex was determined to facilitate tracking of 2 well documented reference points in the pulse wave signals: the maximum of acceleration (MA) and the dicrotic notch (DN) in the acceleration signal (the $1^{\text {st }}$ derivative of the raw LDV-signal). The peaks in the acceleration signal were tracked in the 3 vibrometer signals, relative to the QRS-complex of every heartbeat. Peaks in this signal clearly shift in time when a distance is traveled. The position of the peaks was then related to the traveled distance (measurement locations are aligned on a straight line, with mutual distance of approximately 1 $\mathrm{cm})$. The linear relationship was tested on the peaks from the 3 LDV systems and the slope returned the PWV $\mathrm{VDV}_{\text {LD }}$ using the MA and the DN (see Figure 2). This was done for every heartbeat: only if the coefficient of determination was larger than 0.99 the PWV-value was kept for further analysis. This way, from every patient, between 3 and 54 PWV-values were obtained, with a median of 27 observations. Although the automatic rejection of non-linear relationship is quite conservative, it is a necessary step in the analysis given the complex nature of the signals, and the fact that only 3 points are tracked. Similar rejection procedures are implemented by Hermeling et al. (Hermeling et al. 2009). Of the 52 patients initially present in the study, 4 patients ( 3 male/ 1 female) were excluded because the quality of the data did not meet our standards for further processing. Eventually, 48 patients were considered for further analysis. 


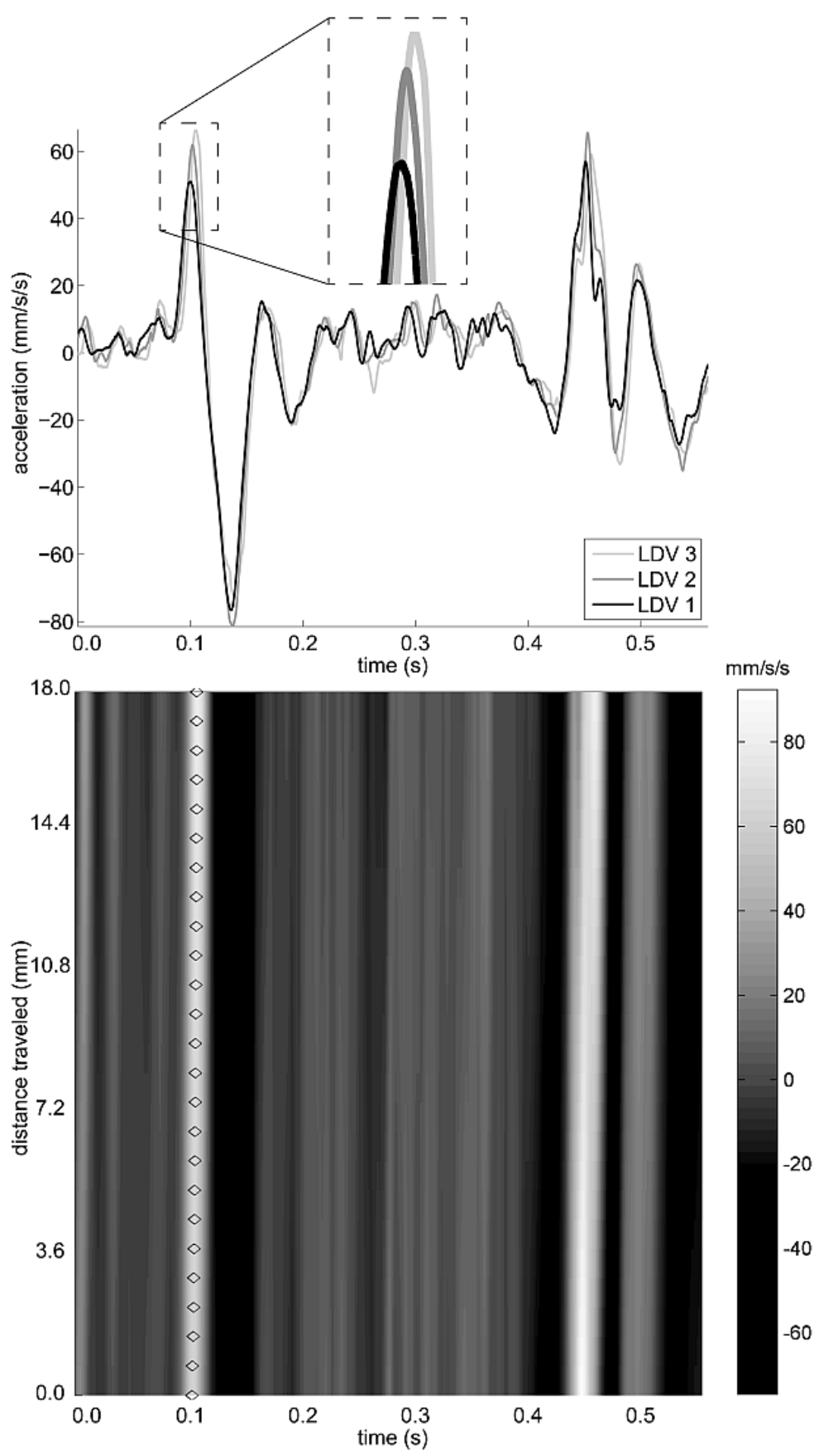

Figure 2: 3 laser Doppler vibrometer systems (LDV 1-3) record the out-of-plane acceleration of the skin on top of the CCA. LDV 1 is aimed at a location closer to the chest, and LDV 3 is aimed closer to the head. In the maximum of acceleration (MA) (left dashed box) a gradual time shift of the signal is seen as the pulse wave travels along the common carotid artery (CCA). In the lower pane, a spatiotemporal rendition of the plot in the upper pane illustrates the propagation of the MA. For illustration purposes, signals of 3 vibrometers on 3 actual measurement locations were resampled to 25 virtual measurement locations. Diamonds indicate the position of the MA (left spree) along the virtual positions. The y-axis indicates position along the CCA, with $18.0 \mathrm{~mm}$ being closer to the head, and $0.0 \mathrm{~mm}$ being closer to the chest. The grey color code indicates amplitude of acceleration. 


\subsubsection{Echography:}

The equipment for US echography was a Prosound Alpha 6 (Hitachi Aloka Medical, Wallington, Connecticut, USA). US echography was performed prior to each LDV-measurement to know the exact position of the CCA and the bifurcation. Additionally, IMT, internal systolic and diastolic diameter $\left(\mathrm{D}_{\text {systolic }}\right.$ and $\left.\mathrm{D}_{\text {diastolic }}\right)$ were assessed. The IMT was measured, using an automated wall-tracking system

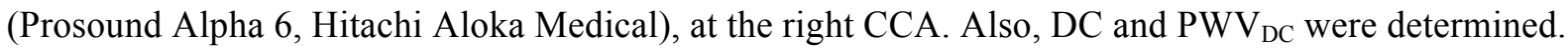
DC is calculated as follows:

$$
D C=\frac{\left(\frac{\left(A_{\text {systolic }}-A_{\text {diastolic }}\right)}{A_{\text {diastolic }}}\right)}{B P_{\text {systolic }-C C A}-B P_{\text {diastolic }-C C A}}
$$

$\mathrm{PWV}_{\mathrm{DC}}$ is calculated using the Bramwell-Hill relationship:

$$
P W V_{D C}=\sqrt{\frac{\left(B P_{\text {systolic-CCA }}-B P_{\text {diastolic-CCA }}\right) * A_{\text {diastolic }}}{\rho_{\text {blood }} *\left(A_{\text {systolic }}-A_{\text {diastolic }}\right)}}=\sqrt{\frac{1}{D C * \rho_{\text {blood }}}}
$$

with $\mathrm{BP}_{\text {systolic-CCA }}$ and $\mathrm{BP}_{\text {diastolic-CCA }}$ being local CCA-pressure derived from Sphygmocor applanation tonometry measurements (Atcor Medical, West Ryde, Australia) instead of brachial cuff pressure; $\mathrm{A}_{\text {systolic }}$ and $\mathrm{A}_{\text {diastolic }}$ being internal cross-sectional area of the CCA-lumen and $\rho_{\text {blood }}$ being blood density. Calibration of the local CCA-pressure was performed by assuming equivalence of mean BP (MBP) (defined as $\left.\mathrm{MBP}=\left(2 * \mathrm{BP}_{\text {diastolic }}+\mathrm{BP}_{\text {systolic }}\right) / 3\right)$ between applanation tonometry and brachial cuff pressure measurements. Additionally, presence of plaques was investigated. All quantitative parameters were recorded 3 times and reported values are the mean of these 3 measurements. All echography recordings were performed by specialized medical personnel.

\subsubsection{Applanation tonometry:}

Applanation tonometry measurements were performed using a Sphygmocor applanation tonometry system, by specialized medical personnel. Measurements were repeated 3 times in each patient, simultaneously with an ECG. cfPWV was calculated using the tangent intersect method and the R-peak of the QRS-complex from the ECG. Distance D between measurement sites was determined with a tapemeasure by measuring distance between CCA and FA. In the cfPWV-calculation, a correction factor of 0.8 was incorporated, accounting for the fact that $\mathrm{D}$ is not the actual distance traveled by the pulse wave due to the aortic arch (Van Bortel et al. 2012):

$$
c f P W V=\frac{D * 0.8}{P T T}
$$

\subsection{Statistics:}

Given the small sample size, and the lack of a priori knowledge of the structure of the relationship between parameters in this population, a non-parametric Spearman correlation analysis was performed on cfPWV, PWV ${ }_{\text {DC }}$ and PWV $\mathrm{V}_{\mathrm{LDV}}$, age, brachial and carotid BP (systolic, diastolic and pulse pressure), CCAdiameter (systolic, diastolic and diameter difference) and IMT. Results were retained at the 99\% confidence level.

A Bland-Altman (BA) assessment was performed on PWV $\mathrm{VCC}_{\mathrm{DC}}$ and $\mathrm{PWV} \mathrm{V}_{\mathrm{LDV}}$, and on cfPWV and $\mathrm{PWV} \mathrm{V}_{\mathrm{LDV}}$. In order to compare this work to other studies, the linear relationship between age and PWV $\mathrm{LDV}_{\mathrm{L}}$ was assessed using a univariate linear regression analysis. 


\section{Results}

Between PWV and physical parameters following significant correlations could be found using nonparametric Spearman correlation analysis: $P W V_{L D V}$ is significantly correlated with age $(R=0.432 ; p<$ $0.005)$ (see Figure $3 \mathrm{a}$, and Table 2$) ; \mathrm{PWV}_{\mathrm{DC}}$ is significantly correlated with $\mathrm{IMT}(\mathrm{R}=0.369, \mathrm{p}<0.01)$ (see Table 2); with $\mathrm{D}_{\text {diastolic }}(\mathrm{R}=0.382 ; \mathrm{p}<0.01)$ (see Table 2) and with $\mathrm{BP}_{\text {systolic-CCA }}(\mathrm{R}=0.504 ; \mathrm{p}<$ 0.001 ) (see Figure $3 \mathrm{f}$ and Table 2).

BA-assessment of $\mathrm{PWV}_{\mathrm{LDV}}$ as compared to cfPWV, shows that the $95 \%$ limits of agreement ranged from $6.95 \mathrm{~m} / \mathrm{s}$ to $0.99 \mathrm{~m} / \mathrm{s}$. cfPWV provides clinically significant higher values, as the level of disagreement includes discrepancies of up to $3.97 \mathrm{~m} / \mathrm{s}$ (see Figure 4a), and $\mathrm{PWV}_{\mathrm{LDV}}$ and $\mathrm{PWV}_{\mathrm{DC}}$ do not correlate (see Figure $4 \mathrm{~b}$ and Table 2). A positive trend can be observed in the BA-plot (see Figure 4a).

BA-assessment of $\mathrm{PWV}_{\mathrm{LDV}}$ as compared to $\mathrm{PWV}_{\mathrm{DC}}$, shows that the $95 \%$ limits of agreement ranged from $0.88 \mathrm{~m} / \mathrm{s}$ to $-1.70 \mathrm{~m} / \mathrm{s}$. The two methods provide measures in the same order of magnitude because the level of disagreement includes discrepancies of up to $-0.41 \mathrm{~m} / \mathrm{s}$ (see Figure $4 \mathrm{c}$ ), however, PWV $\mathrm{LDV}_{\text {and }}$ $\mathrm{PWV}_{\mathrm{DC}}$ do not correlate (see Figure $4 \mathrm{~d}$ and Table 2). A positive trend can be observed in the BA-plot (see Figure 4d).

According to a univariate linear regression analysis, an age dependent increase in $\mathrm{PWV}_{\text {LDV }}$ of $0.06 \mathrm{~m} / \mathrm{s}$ per additional year $(\mathrm{R}=0,173 ; \mathrm{p}=0.002)$ can be observed (see Figure $3 \mathrm{a}$ ).
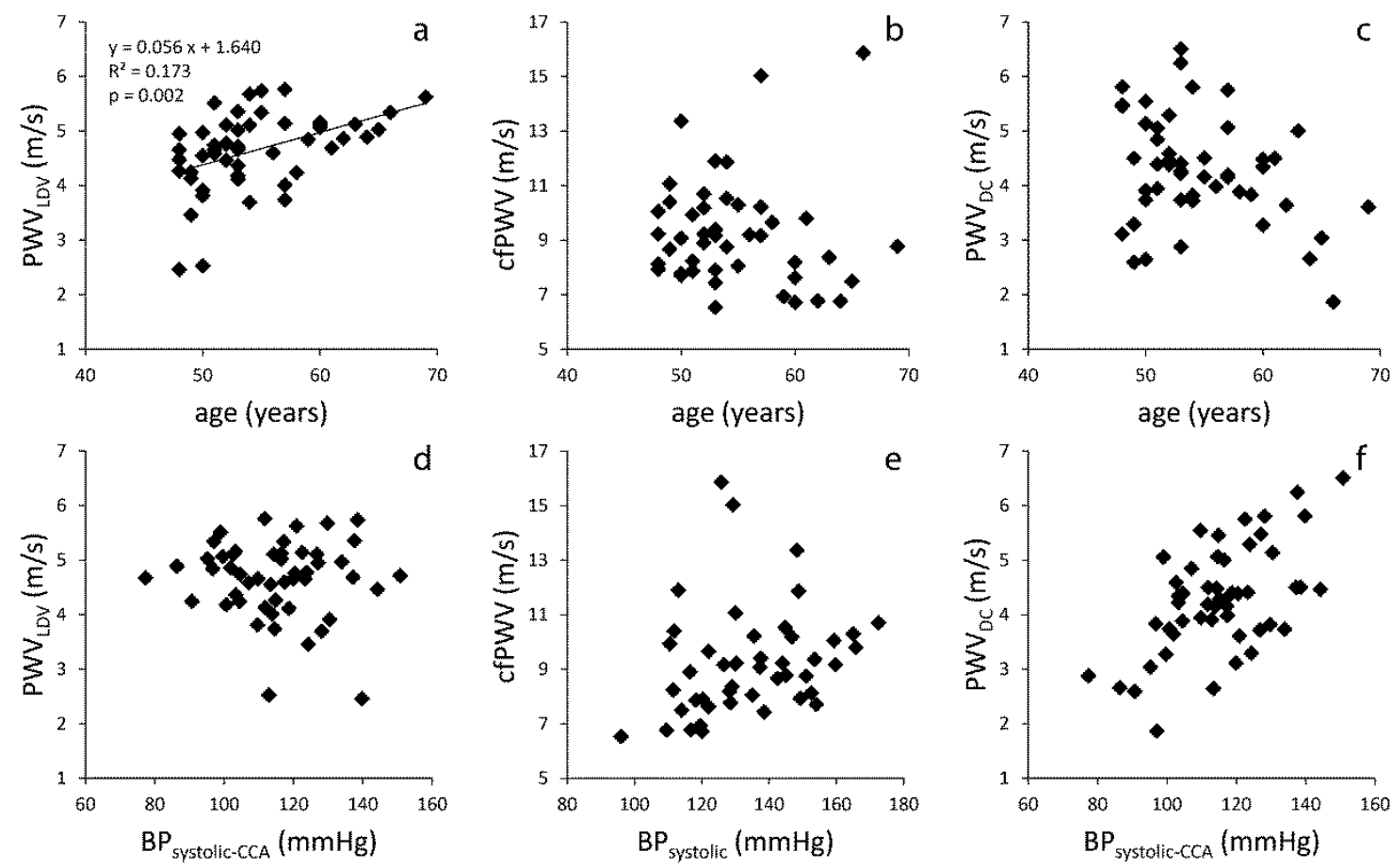

Figure 3: Correlation plot illustrating the relation between three different indices of arterial stiffness (pulse wave velocity measured (PWV) with laser Doppler vibrometry (LDV), tonometry and distensibility coefficient (DC) respectively, or $\mathrm{PWV}_{\mathrm{LDV}}$, cfPWV and $\mathrm{PWV}_{\mathrm{DC}}$ ) and the main determinants of arterial stiffness age and brachial $\left(\mathrm{BP}_{\text {systolic }}\right)$ or common carotid artery $\left(\mathrm{BP}_{\text {systolic-CCA }}\right)$ systolic blood pressure (BP). The outcome of a univariate linear regression analysis is shown in (a). 

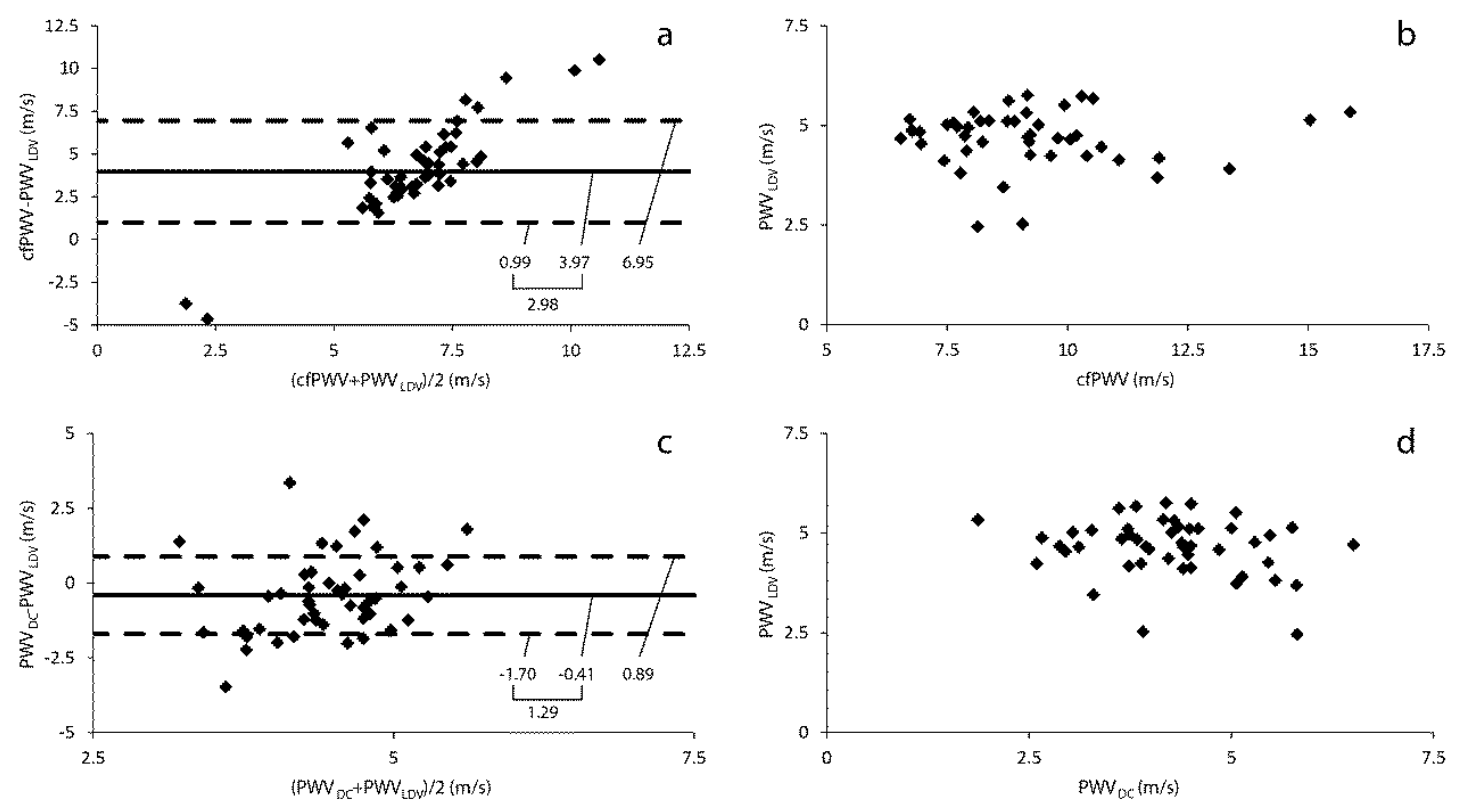

Figure 4: Bland-Altman (BA) assessment of $\mathrm{PWV}_{\mathrm{LDV}}$ (pulse wave velocity (PWV) measured with laser Doppler vibrometry (LDV)) as compared to cfPWV (PWV measured with tonometry) (a) and of PWV $\mathrm{LDV}_{\text {. }}$ as compared to $\mathrm{PWV}_{\mathrm{DC}}$ (PWV measured with the distensibility coefficient (DC)) (c). Limits of agreement, and the level of discrepancy is indicated on the graph; correlation plot illustrating the relation between cfPWV and PWV $\mathrm{LDV}_{(\mathrm{b}) \text { and between } \mathrm{PWV}_{\mathrm{DC}} \text { and } \mathrm{PWV}} \mathrm{WDV}_{\mathrm{L}}(\mathrm{d})$. 
Table 2: Correlation Matrix

\begin{tabular}{|c|c|c|c|c|c|c|c|c|c|c|}
\hline & $\stackrel{0}{g}$ & $\sum_{\substack{\infty \\
\infty}}^{\nabla}$ & $\sum_{\substack{E \\
<}}^{\nabla}$ & $\underset{\substack{0 \\
0}}{D}$ & 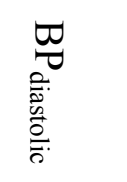 & 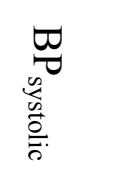 & 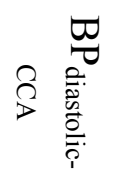 & 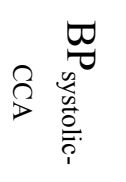 & $\underset{7}{3}$ & \\
\hline \multirow{2}{*}{ cfPWV } & & 0.212 & -0.069 & -0.150 & 0.160 & 0.329 & 0.176 & 0.323 & 0.233 & $\mathrm{CC}$ \\
\hline & & 0.144 & 0.636 & 0.303 & 0.272 & 0.021 & 0.226 & 0.023 & 0.107 & sig. \\
\hline \multirow{2}{*}{$\mathrm{PWV}_{\mathrm{DC}}$} & & & -0.129 & -0.314 & $\underline{0.368}$ & 0.348 & 0.214 & $\underline{0.518}$ & $\underline{0.382}$ & $\mathrm{CC}$ \\
\hline & & & 0.378 & 0.028 & $\underline{0.009}$ & 0.014 & 0.141 & $\underline{0.000}$ & $\underline{0.007}$ & sig. \\
\hline \multirow{2}{*}{$P W V_{L D V}$} & & & & $\underline{0.432}$ & 0.140 & -0.022 & 0.091 & -0.035 & -0.330 & $\mathrm{CC}$ \\
\hline & & & & 0.002 & 0.339 & 0.882 & 0.533 & 0.813 & 0.021 & sig. \\
\hline \multirow{2}{*}{ Age } & & & & & -0.106 & -0.220 & -0.121 & -0.256 & -0.278 & $\mathrm{CC}$ \\
\hline & & & & & 0.469 & 0.129 & 0.410 & 0.076 & 0.053 & sig. \\
\hline \multirow{2}{*}{$\mathrm{BP}_{\text {diastolic }}$} & & & & & & $\underline{0.765}$ & $\underline{0.922}$ & $\underline{0.768}$ & 0.063 & $\mathrm{CC}$ \\
\hline & & & & & & $\underline{0.000}$ & $\underline{0.000}$ & $\underline{0.000}$ & 0.669 & sig. \\
\hline \multirow{2}{*}{$\mathrm{BP}_{\text {systolic }}$} & & & & & & & 0.847 & $\underline{0.925}$ & 0.076 & $\mathrm{CC}$ \\
\hline & & & & & & & $\underline{0.000}$ & $\underline{0.000}$ & 0.604 & sig. \\
\hline \multirow{4}{*}{$\begin{array}{l}\mathrm{BP}_{\text {diastolic- }} \\
\quad \mathrm{CCA} \\
\mathrm{BP}_{\text {systolic- }} \\
\mathrm{CCA}\end{array}$} & & & & & & & & $\underline{0.752}$ & 0.031 & $\mathrm{CC}$ \\
\hline & & & & & & & & $\underline{0.000}$ & 0.831 & sig. \\
\hline & & & & & & & & & 0.125 & $\mathrm{CC}$ \\
\hline & & & & & & & & & 0.392 & sig. \\
\hline
\end{tabular}

Table 2: correlation matrix of all evaluated parameters. Correlations were retained at the $99 \%$ significance level, and retained correlations are indicated in bold and underlined. Abbreviations: CC (Spearman correlation coefficient); sig. (significance level); $\mathrm{BP}_{\text {systolic/diastolic }}$ (brachial systolic/diastolic blood pressure); $\mathrm{BP}_{\text {systolic-CCA/diastolic-CCA }}$ (CCA systolic/diastolic blood pressure); IMT (intima-media thickness); $\mathrm{PWV}_{\mathrm{DC}}$ (PWV derived from DC); cfPWV (PWV measured with applanation tonometry); PWV $\mathrm{LDV}_{\text {(PWV }}$ measured with LDV using the MA).

\section{Conclusions}

\subsection{Comparison with previous research of CCA-stiffness:}

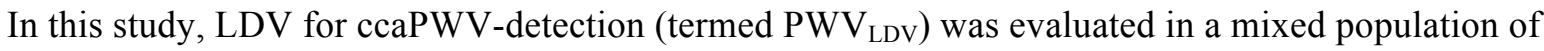
patients at risk for increased arterial stiffness.

Only a handful of studies assess this ccaPWV non-invasively in patients, using such methods as MRI (Kröner et al. 2014; Hardy et al. n.d.), pulse wave imaging (PWI) (Hermeling et al. 2009; Luo et al. 2012) and other US systems (Benthin et al. 1991; Eriksson et al. 2002) and - recently - using LDV (Campo et al. 2014).

PWV can be measured accurately and in controlled conditions using MRI. However, the assessment of ccaPWV is challenging due to the need for high temporal and spatial resolution. Accordingly, there is only scarce literature describing ccaPWV-assessment with MRI: One conference proceeding described PWVmeasurements in the carotids in three healthy volunteers using velocity-encoded MRI (VE-MRI) (Hardy et al. n.d.). Another used VE-MRI at 2 locations along the CCA. According to the latter, ccaPWV was found 
to be $5.7 \pm 1.0$ in volunteers younger than 30 , and $6.9 \pm 1.5$ in volunteers older than 45 . For the volunteers older than 45, an increase of $0.09 \mathrm{~m} / \mathrm{s}$ per incremental year was found (Kröner et al. 2014).

PWI was used to detect ccaPWV by Luo et al. (Luo et al. 2012) and Hermeling et al. (Hermeling et al. 2009). Using the DN as a reference point, Hermeling et al. found ccaPWV to be $5.0 \pm 0.7 \mathrm{~m} / \mathrm{s}$ in the young and $7.5 \pm 1.5 \mathrm{~m} / \mathrm{s}$ in the older participants. Luo et al. found ccaPWV to be $4.5 \pm 0.4 \mathrm{~m} / \mathrm{s}$ in the young. Other US methods were used as well for PWV-detection by Benthin et al. (Benthin et al. 1991) and Eriksson et al. (Eriksson et al. 2002). The latter measured one subject of 30 years old and rendered a value of $8.3 \pm 0.6 \mathrm{~m} / \mathrm{s}$.

In this study using LDV, a group of subjects at cardiovascular risk is assessed. The subjects are older than 45 and $P W V_{L D V}$ in this population is $4.6 \pm 0.7 \mathrm{~m} / \mathrm{s}$. $\mathrm{PWV}$ LDV is found to correlate positively with age with an increase in $P W V_{L D V}$ of $0.06 \mathrm{~m} / \mathrm{s}$ per incremental year (see Figure 3a). Although studies directly evaluating the effect of skin elasticity on ccaPWV are non-existent, it can be hypothesized that the effect of skin elasticity on $\mathrm{PWV}_{\mathrm{LDV}}$ is not becoming more important with age as the skin becomes more loose with time (Robert et al. 1988; Henry et al. 1997). Therefore, it can be assumed that the increase of carotid stiffness over age is mainly due to mechanical changes on the carotid level. In conclusion, the found $\mathrm{PWV}_{\mathrm{LDV}}$ values, and the increase of $\mathrm{PWV} \mathrm{V}_{\mathrm{LDV}}$ over age are well in line with ccaPWV-values from literature as measured with other modalities, although presented $P W V_{L D V}$ values can be considered more typical for a younger population.

\subsection{Discrepancies with previous observations:}

However, in contrast to our expectations, no relation could be found with the other indices of arterial stiffness or with BP. Several explanations can be found for the latter observation.

Although cfPWV is considered the golden standard for arterial stiffness determination, it is still a very different parameter than CCA-stiffness for reasons mentioned in the introduction. It can be assumed that in this population at risk, the differentiation between central and peripheral arteries is even more reflected in the cfPWV-value, affecting its sensitivity. Moreover, the very heterogenic, small patient population, in combination with a diverse array of medication in every individual might introduce several differential effects between carotid-femoral and CCA trajectories. This is reflected in the lack of - elsewhere reported - correlation between cfPWV and DC, IMT and age.

A lack of correlation of ccaPWV with DC is reported by Hermeling et al., who suggests using the DN instead of the MA. However, the DN could not be assessed unambiguously in a substantial portion of the data. This is not surprising - as a possible limitation of the technique - LDV is very sensitive to all kinds of velocity components contributing to the assessed skin surface. These components can originate from breathing and other involuntary muscle contractions, pulsations from nearby arteries and even veins (Mignanelli et al. 2014), and vibrations from the building and the measurement setup itself. We kept the rejection procedures for data quite strict to account for the variability in the biological samples and the many artifacts from aforementioned sources, such that most DN values were rejected. Several factors can

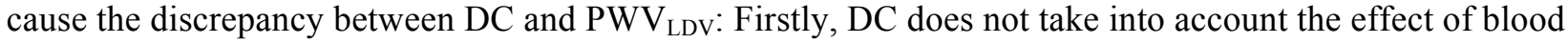
velocity which is known to be affected by age (Schmidt-Trucksäss et al. 1999). This can cause a substantial discrepancy between PWV and DC (Parker \& Jones 1990) especially in arteries with low PWV and high blood velocity such as is the case in the CCA, particularly during systole when blood velocities can be as high as $1 \mathrm{~m} / \mathrm{s}$ (Segadal \& Matre 1987). The latter difference in blood velocity might be reflected in the systematic bias as found with theoretical $\mathrm{PWV}_{\mathrm{DC}}$ based on the $\mathrm{DC}$ : $\mathrm{PWV}_{\mathrm{LDV}}$ is systematically higher with $0.41 \mathrm{~m} / \mathrm{s}$. Secondly, the DC is calculated using the change in pressure and area over the whole 
cardiac cycle. The arterial non-linear pressure-area relation will introduce an overestimation of the DC and consequently an underestimation of $\mathrm{PWV}_{\mathrm{DC}}$ as possibly reflected in the data (Pruett et al. 1988). Finally, no simultaneous pressure and distension measurements were performed, possibly influencing the $\mathrm{PWV}_{\mathrm{DC}}$. A relation with IMT could neither be found. Although moderate increases in IMT are known to correlate with arterial stiffness, in the elderly and in atherosclerosis patients, the IMT is rather related to atherosclerosis (Bots et al. 1997). Since the population assessed in this study consists entirely of elder patients with atherosclerosis, it can be hypothesized that IMT-values do rather represent atherosclerosis, and only moderately relate to arterial stiffness.

Finally, PWV $\mathrm{LDV}_{\mathrm{LV}}$ does not relate to BP, which is believed to be a major determinant of cfPWV. As cfPWV does not relate to $\mathrm{PWV}_{\mathrm{LDV}}$, and a lack of correlation of $\mathrm{PWV}$ with $\mathrm{BP}$ is also reported by Hermeling et al. this is not surprising. We hypothesize that this might be due to the fact that we use the MA which is more susceptible to reflections, affecting its precision and accuracy, in combination with the patients' medication that affects BP, arterial stiffness, and the relation between those two. Additionally, Safar et al. indicate that BP is not the only factor of arterial stiffening. Hyperlipidemia, diabetes mellitus, tobacco consumption and other factors present in our population affect arterial stiffness as well (Safar et al. 2000).

\subsection{General conclusion:}

In conclusion, only $\mathrm{PWV}_{\mathrm{DC}}$ gave direct information about CCA-stiffness with high precision, as it reflected systolic BP, IMT and diastolic diameter. However, this parameter is based on DC, and as such, blood velocity is not taken into account. It can be argued that the latter parameter needs to be taken into account since not only the DC but also ground-truth PWV is believed to be important in the etiology of hypertrophy of the heart.

Relations found with $\mathrm{PWV} \mathrm{V}_{\mathrm{DC}}$ could not be reproduced using $\mathrm{PWV} \mathrm{V}_{\mathrm{LDV}}$. However, it is reasonable to conclude that $P W V_{L D V}$ does bear relevant information about the arterial system. Higher $\mathrm{PWV}_{\mathrm{LDV}}$ comes with higher age as confirmed in other studies assessing ccaPWV and PWV in general; and age is one of the most important determinants of arterial stiffness regardless of the index used. We hypothesize that blood velocity, in combination with a patients' medication, other influences and artifacts from various sources, are at least to a certain extent masking the effects of arterial parameters and BP on PWV $\mathrm{LDV}_{\text {, and }}$ some of the expected relations might even be non-existent in this small heterogenic population.

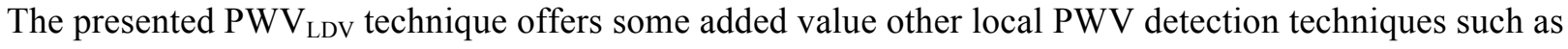
US and MRI: the LDV device can be made compact and handheld, and no bulky ultrasound or MRI equipment is needed. Also, the readout of the measurement is direct with no laborious post-processing of huge datasets, such that results can enter the statistics easily. This makes introduction in the clinical practice feasible, and also allows for large scale studies. Finally, the technique is non-contact, such that any interference with artery mechanics during measurements is absent.

In future work, blood velocity measurements of the CCA should be included to rule out the effect of this specific parameter. Moreover, a more uniform or a larger patient population should be used. Finally, as a true reference method, PWI or MRI should be incorporated in future studies in order to compare PWV $\mathrm{LDV}_{\mathrm{L}}$ with a real local PTT method for PWV-detection.

\section{Acknowledgements}


We thank prof. Patrick Segers from the Biofluid, Tissue and Solid Mechanics for Medical Applications group (bioMMeda, Ghent University) for his professional advice, and the Research Foundation Flanders (FWO) for financial support.

\section{Disclosure}

The authors report that there is no conflict of interest.

\section{References}

Ahlgren, A.R. et al., 1997. Stiffness and diameter of the common carotid artery and abdominal aorta in women. Ultrasound in medicine \& biology, 23(7), pp.983-8.

Benetos, A. et al., 1993. Arterial alterations with aging and high blood pressure. A noninvasive study of carotid and femoral arteries. Arteriosclerosis and thrombosis : a journal of vascular biology / American Heart Association, 13(1), pp.90-7.

Benthin, M. et al., 1991. Calculation of pulse-wave velocity using cross correlation--effects of reflexes in the arterial tree. Ultrasound in medicine \& biology, 17(5), pp.461-9.

Blacher, J. et al., 1998. Carotid arterial stiffness as a predictor of cardiovascular and all-cause mortality in end-stage renal disease. Hypertension, 32(3), pp.570-4.

Van Bortel, L.M. et al., 2012. Expert consensus document on the measurement of aortic stiffness in daily practice using carotid-femoral pulse wave velocity. Journal of hypertension, 30(3), pp.445-8.

Bots, M.L., Hofman, A. \& Grobbee, D.E., 1997. Increased common carotid intima-media thickness. Adaptive response or a reflection of atherosclerosis? Findings from the Rotterdam Study. Stroke; $a$ journal of cerebral circulation, 28(12), pp.2442-7.

Boutouyrie, P. et al., 1995. Common Carotid Artery Stiffness and Patterns of Left Ventricular Hypertrophy in Hypertensive Patients. Hypertension, 25(4), pp.651-659.

Campo, A. et al., 2014. Non-invasive technique for assessment of vascular wall stiffness using laser Doppler vibrometry. Measurement Science and Technology, 25(6), p.065701.

Cooper, N.P., 1999. An improved heterodyne laser interferometer for use in studies of cochlear mechanics. Journal of Neuroscience Methods, 88(1), pp.93-102.

Eriksson, A. et al., 2002. Arterial pulse wave velocity with tissue Doppler imaging. Ultrasound in medicine \& biology, 28(5), pp.571-80.

Hardy, C. et al., MRI determination of pulse wave velocity in the carotid arteries. Proc 16th Annual Meeting ISMRM, Toronto; 2008.

Henry, F. et al., 1997. Age-Related Changes in Facial Skin Contours and Rheology. Journal of the American Geriatrics Society, 45(2), pp.220-222.

Hermeling, E. et al., 2007. Measurement of local pulse wave velocity: effects of signal processing on precision. Ultrasound in medicine \& biology, 33(5), pp.774-81.

Hermeling, E. et al., 2009. The dicrotic notch as alternative time-reference point to measure local pulse wave velocity in the carotid artery by means of ultrasonography. Journal of hypertension, 27(10), pp.2028-35.

Hirata, K., Momomura, S. \& O’Rourke, M.F., 2010. Carotid flow augmentation as a risk for small vessel 
disease of the brain. American journal of hypertension, 23(9), p.932; author reply 933.

Huybrechts, S.A.M. et al., 2011. Carotid to femoral pulse wave velocity: a comparison of real travelled aortic path lengths determined by MRI and superficial measurements. Journal of hypertension, 29(8), pp.1577-82.

Kimoto, E. et al., 2003. Preferential Stiffening of Central Over Peripheral Arteries in Type 2 Diabetes. Diabetes, 52(2), pp.448-452.

Kröner, E.S.J. et al., 2014. Pulse wave velocity and flow in the carotid artery versus the aortic arch: effects of aging. Journal of magnetic resonance imaging: JMRI, 40(2), pp.287-93.

Länne, T. et al., 1994. Differences in mechanical properties of the common carotid artery and abdominal aorta in healthy males. Journal of vascular surgery, 20(2), pp.218-25.

Laurent, S. et al., 2003. Aortic stiffness is an independent predictor of fatal stroke in essential hypertension. Stroke; a journal of cerebral circulation, 34(5), pp.1203-6.

Laurent, S. et al., 2006. Expert consensus document on arterial stiffness : methodological issues and clinical applications. Stroke, pp.2588-2605.

Leone, N. et al., 2008. Distension of the carotid artery and risk of coronary events: The three-city study. Arteriosclerosis, Thrombosis, and Vascular Biology, 28(7), pp.1392-1397.

Liao, D. et al., 1999. Arterial stiffness and the development of hypertension. The ARIC study. Hypertension, 34(2), pp.201-6.

Luo, J., Li, R. \& Konofagou, E., 2012. Pulse Wave Imaging (PWI) of the human carotid artery: An in vivo feasibility study. The Journal of the Acoustical Society of America, 131(4), pp.3289-3289.

De Melis, M. et al., 2008. A noncontact approach for the evaluation of large artery stiffness: a preliminary study. American journal of hypertension, 21(12), pp.1280-3.

Mignanelli, L. et al., 2014. Medical diagnosis of the cardiovascular system on the carotid artery with IR laser Doppler vibrometer. In 11th International Conference on Vibration Measurements by Laser and Noncontact Techniques, AIVELA 2014. pp. 313 - 322.

Nagai, Y. et al., 1999. Carotid arterial stiffness as a surrogate for aortic stiffness: relationship between carotid artery pressure-strain elastic modulus and aortic pulse wave velocity. Ultrasound in medicine \& biology, 25(2), pp.181-8.

Paini, A. et al., 2006. Carotid and aortic stiffness: determinants of discrepancies. Hypertension, 47(3), pp.371-6.

Parker, K.H. \& Jones, C.J., 1990. Forward and backward running waves in the arteries: analysis using the method of characteristics. Journal of biomechanical engineering, 112(3), pp.322-6.

van Popele, N.M. et al., 2001. Association between arterial stiffness and atherosclerosis: the Rotterdam Study. Stroke; a journal of cerebral circulation, 32(2), pp.454-60.

Pruett, J.D., Bourland, J.D. \& Geddes, L.A., 1988. Measurement of pulse-wave velocity using a beatsampling technique. Annals of biomedical engineering, 16(4), pp.341-7.

Robert, C., Lesty, C. \& Robert, A.M., 1988. Ageing of the Skin: Study of Elastic Fiber Network Modifications by Computerized Image Analysis. Gerontology, 34(5-6), pp.291-296.

Safar, M.E. et al., 2000. Stiffness of carotid artery wall material and blood pressure in humans: application to antihypertensive therapy and stroke prevention. Stroke; a journal of cerebral circulation, 31(3), pp.782-90.

Schmidt-Trucksäss, A. et al., 1999. Structural, functional, and hemodynamic changes of the common carotid artery with age in male subjects. Arteriosclerosis, thrombosis, and vascular biology, 19(4), 
pp.1091-7.

Segadal, L. \& Matre, K., 1987. Blood velocity distribution in the human ascending aorta. Circulation, 76(1), pp.90-100.

Simon, A., Megnien, J.-L. \& Chironi, G., 2010. The value of carotid intima-media thickness for predicting cardiovascular risk. Arteriosclerosis, thrombosis, and vascular biology, 30(2), pp.182-5.

van Sloten, T.T. et al., 2014. Local stiffness of the carotid and femoral artery is associated with incident cardiovascular events and all-cause mortality: the Hoorn study. Journal of the American College of Cardiology, 63(17), pp.1739-47.

Toprak, A. et al., 2009. Relation of pulse pressure and arterial stiffness to concentric left ventricular hypertrophy in young men (from the Bogalusa Heart Study). The American journal of cardiology, 103(7), pp.978-84.

Tran, L.T.T., Park, H. \& Kim, H., 2012. Is the Carotid Intima-Media Thickness Really a Good Surrogate Marker of Atherosclerosis? Journal of Atherosclerosis and Thrombosis, 19, pp.680-690.

Tsamis, A., Krawiec, J.T. \& Vorp, D.A., 2013. Elastin and collagen fibre microstructure of the human aorta in ageing and disease: a review. Journal of the Royal Society, Interface / the Royal Society, 10(83), p.20121004.

Tsivgoulis, G. et al., 2006. Common carotid arterial stiffness and the risk of ischaemic stroke. European journal of neurology: the official journal of the European Federation of Neurological Societies, 13(5), pp.475-81.

Weisz, S.H. et al., 2014. Carotid artery and aortic stiffness evaluation in aortic stenosis. Journal of the American Society of Echocardiography, 27, pp.385-392. 